JDAL

5,2

\section{4}

Received 20 September 2021 Revised 26 October 2021 Accepted 26 October 2021

\title{
Developing a resilient, robust and efficient supply network in Africa
}

\author{
Jade F. Preston and Bruce A. Cox \\ Department of Operational Sciences, Air Force Institute of Technology, \\ Wright-Patterson AFB, Ohio, USA \\ Paul P. Rebeiz \\ o9 Solutions Inc., Dallas, Texas, USA, and \\ Timothy W. Breitbach \\ Department of Operational Sciences, Air Force Institute of Technology, \\ Wright-Patterson AFB, Ohio, USA
}

\begin{abstract}
Purpose - Supply chains need to balance competing objectives; in addition to efficiency, supply chains need to be resilient to adversarial and environmental interference and robust to uncertainties in long-term demand. Significant research has been conducted designing efficient supply chains and recent research has focused on resilient supply chain design. However, the integration of resilient and robust supply chain design is less well studied. The purpose of the paper is to include resilience and robustness into supply chain design.

Design/methodology/approach - The paper develops a method to include resilience and robustness into supply chain design. Using the region of West Africa, which is plagued with persisting logistical issues, the authors develop a regional risk assessment framework and then apply categorical risk to the countries of West Africa using publicly available data. A scenario reduction technique is used to focus on the highest risk scenarios for the model to be tractable. Next, the authors develop a mathematical model leveraging this framework to design a resilient supply network that minimizes cost while ensuring the network functions following a disruption. Finally, the authors examine the network's robustness to demand uncertainty via several plausible emergency scenarios.

Findings - The authors provide optimal sets of transshipment hubs with varying counts from 5 through 15 hubs. The authors determine there is no feasible solution that uses only five transshipment hubs. The authors' findings reinforce those seven transshipment hubs - the solution currently employed in West Africa - is the cheapest architecture to achieve resilience and robustness. Additionally, for each set of feasibility transshipment hubs, the authors provide connections between hubs and demand spokes.

Originality/value - While, at the time of this research, three other manuscripts incorporated both resilience and robustness of the authors' research unique solved the problem as a network flow instead of as a set covering problem. Additionally, the authors establish a novel risk framework to guide the required amount of redundancy, and finally the out research proposes a scenario reduction heuristic to allow tractable exploration of 512 possible demand scenarios.
\end{abstract}

Keywords Network optimization, Supply chain design, Resilient networks, Robust networks, Scenario analysis

Paper type Research paper

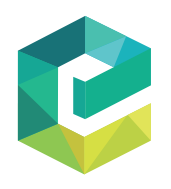

\footnotetext{
(C) In accordance with section 105 of the US Copyright Act, this work has been produced by a US government employee and shall be considered a public domain work, as copyright protection is not available. Published in Journal of Defense Analytics and Logistics. Published by Emerald Publishing Limited.

The authors would like to thank the editors and reviewers for excellent suggestions leading to substantive improvements in readability and content.

Disclaimer: The views expressed in this article are those of the authors and do not reflect the official policy or position of the United States Air Force, the Department of Homeland Security, the Department of Defense, or the United States Government.
} Logistics
Vol. 5 No. 2, 2021 pp. $224-241$ Emerald Publishing Limited 2399-6439

DOI 10.1108/JDAL-09-2021-0006 


\section{Introduction}

The Ebola outbreak and the rise of terrorist organizations such as Boko Haram and the Islamic State in Africa have given Western Africa a recent place of prominence on the global stage. In conjunction with this rise in global awareness, multiple agencies across the USA Government - from the Department of Defense to the Center for Disease Control and Department of State - have increased their presence in the region. Western Africa, encompassing 18 countries roughly the size of the continental USA, has become the region in Africa of highest logistical concern for the USA Government. With this increased presence comes a critical need for reliable logistics support.

A key challenge facing the USA, the international community and commercial companies establishing operations in Africa - especially Western Africa given the number of countries, their expansiveness and the infrastructure condition - is the development of a robust, resilient and efficient supply chain network $(\mathrm{SCN})$. Adding to the complexity of this issue is the fact that demand densities across the region are low and highly variable. Historically, the USA Government supported humanitarian, military or other operations through the use of $a d$ hoc and contingency-scheduled air mobility missions. However, this has become costly and unreliable. The absence of an efficient, resilient and robust aerial distribution system hinders the USA and international community's ability to respond to humanitarian crises and help local governments combat terrorism.

In this paper, we examine a hub and spoke distribution network currently in use by the USA Government in which mixed commodities of material, originating in the USA or Europe, flow into a main hub on the West African coast. In addition to the main hub, there are currently 6 transshipment nodes spread throughout the region that support up to 54 additional potential demand locations. We allow for a multi-modal distribution network in which products can be transported by air, ground or water, but the primary mode remains air due to large distances and difficult terrain. Low concentrations of USA and allied personnel in the region result in low-demand levels with a high variability and occasional demand spikes due to regional events.

This manuscript's primary contributions are as follows: First, we propose a risk analysis framework that codifies the risk of supply chain disruptions associated with the region's environmental challenges, natural barriers and internal conflicts. We employ a scenario reduction technique to design the network in accordance with the highest-risk scenarios. We then use the outcome of this risk framework to parameterize a two-stage stochastic optimization network flow model. The resulting network is both resilient to disruption and robust to uncertain future demand. To our knowledge, this is the first time a decision-analysis framework is used in conjunction with the development of a resilient and robust supply chain design.

The paper is structured as follows. Section 2 presents a literature review of SCNs design and codifies meanings for disruptions, resilience and robustness in the context of graph theory. Section 3 outlines the risk analysis framework and the underlying mathematical model parameters, inputs and formulation. Section 4 outlines the disruption scenarios and model variations considered, and then it analyzes several model instantiations. Section 5 summarizes research findings and recommendations. Section 6 concludes by summarizing our research and providing future research directions.

\section{Resilient, robust and efficient supply chain network designs}

Developing a supply chain for West Africa is a SCN design problem, which is a subset of the facility location problem [See, for instance, Mirchandani and Francis (1990) and Daskin (2011) for excellent treatments of the facility location problem]. Specifically, the SCN design problem models the supply chain as a network via graph theory (Wagner and Neshat, 2010). Nodes in
Developing a resilient, robust supply chain 
JDAL 5,2

226 the graph represent the locations of interest (i.e. suppliers, warehouses and or customers) and the graph's arcs represent the transportation routes between these locations.

\subsection{Supply chain efficiency}

Supply chain efficiency consists of attaining the supply chain's goals with minimum resources, thus achieving cost-related advantages (Borgström, 2005). Efficiency is thus naturally one of the dominant considerations in developing SCNs. Jackson and Wolinsky (1996) showed that efficient networks are either complete graphs, star graphs or graphs with no links. Heydari et al. (2015) showed that any two nodes in an efficient network have a maximum distance of two connections between them. If this is not the case, the routes can be restructured to obtain a network that achieves similar or better results.

Hub-and-spoke networks are a type of efficient networks. Under a hub-and-spoke structure, demand locations (spokes) are all connected to warehouses (hubs). Yang et al. (2017) discuss the efficiency of this type of design in which hubs are situated to minimize the connection length between spokes. This corresponds to placing hubs in a centralized location, which minimizes the number of hubs. As such, one can conclude that the transshipment nodelocation problem and the hub-and-spoke design problems both aim to achieve network efficiency. Although efficient networks provide great benefit by reducing the number of components while simultaneously satisfying demand, such networks pose notable challenges.

Efficient networks are not always stable, and they are not always able to alter connections without damaging function (i.e. be resilient to disruption) (Jackson and Wolinsky, 1996). In an efficient network, removal of a link or of a node and its links could have serious ramifications, potentially sabotaging any productivity gained by using an efficient network. This motivates the consideration of network resilience. Mensah and Merkuryev (2014) and Sheffi and Rice (2005) define resilience as the network's capacity to return to its original state after disruption or to mitigate disruption of network flow entirely. Hutchison and Sterbenz (2018) define resilience as a network's ability to maintain its service level during disruptions. However, both of these definitions fail to explicitly define what is meant by a "disruption." Indeed, Kim et al. (2015) notes this exact problem and provides 16 different academic definitions for a disruption.

\subsection{Supply chain resilience}

Berdica (2002) provides a seminal work linking transportation network vulnerability and transportation network resilience. Specifically, they introduce resilience in terms of a system's dynamic stability. Tying the concepts of a disruptive event's probability of occurrence and consequence to system resilience, more recently, Mattsson and Jenelius (2015) summarize the state of the art in research on transportation vulnerability and its linkage to network resilience. Matisziw et al. (2009) also explore network resilience by exploring SCN vulnerability through the simulation of disruptions. Similarly, Muckensturm and Longhorn (2019) assess military theater distribution vulnerability via an NP-hard graph problem which they solve heuristically.

Chowdhury and Quaddus (2017) define three dimensions for supply chain resilience as follows: proactive, reactive and supply chain design quality. Proactive resilience relates to design choices made $a$ priori such as increasing capacity or redundancy. Reactive resilience deals with the ability of a supply chain to recover from a disruption, and supply chain design quality deals with node density, SCN complexity and node criticality. For the purposes of this research, we utilize a graph-theoretic definition for resilience similar to that used by Kim et al. (2015). Specifically, we state that a disruption is any action (natural or man-made) that removes an arc or a node from the network. In the context of Chowdhury and Quaddus (2017), 
our definition of resilience falls under both the proactive and supply chain design-quality dimensions, as our resilience metric will motivate the creation of redundant supply availability (i.e. multiple allocation Campbell (1996)). This is a rather binary definition, as in the real world partial degradation is more likely, but it establishes a conservative design. We thus define a node's resilience as the number of arcs that can be removed from the network before flow constraints prevent demand from being met at this node.

Jackson and Wolinsky (1996) identify a tetrahedron as a stable network, as all links are connected to one another. Thus, even with removal of an arc or node the network preserves connectivity between the nodes. Clearly, then networks can become more resilient by adding nodes or more arcs. Zhalechian et al. (2018) argue that adding resilience to their huband-spoke model could be achieved by connecting each node to multiple hubs. They construct a fortified hub-and-spoke network, which resulted in a resilient network. This network has built-in proactive and reactive capabilities, protecting it from natural disasters, terrorist attacks and internal network problems. Ng et al. (2018) address networkrisk management by classifying nodes and arcs based on their geographic characteristics and were able to mitigate the risk of network delays by assessing each node's ease of access and its suitability as a hub or spoke. Sadghiani et al. (2015) use a set-covering approach to ensure demand locations are covered by multiple suppliers based on characteristics of the demand locations in order to increase resilience. An et al. (2015) propose a reliable hub-andspoke network through the creation of hub backups and alternative flow routes. Similarly, Rostami et al. (2018) ensure resilience through the assignment of backup hubs. However, as compared with this paper, both Rostami and An et al. solve an assignment problem vs a network flow. Reggiani et al. (2015) address transportation system resilience through the lens of spatial complexity analysis, specifically through the concepts of connectivity and accessibility. Such approaches focus on ensuring SCN resilience by adding routes and/or nodes that preserve all locations' ability to meet their demand. These network additions are targeted at the location level. Such approaches are aligned with the technique we present in section 3 .

\subsection{Supply chain robustness}

Network robustness is an additional topic relevant to SCN design, which is often confounded with resilience. Yang et al. (2017) point out that designing a network corresponds to a long-term strategic decision under a dynamic and changing environment and that due to variations in the network's geospatial inputs, a robust network could be required rather than an efficient network. Klibi et al. (2010) define robustness as "the quality of a SCN to remain effective for all plausible futures." In general, robustness is a network's ability to function "well" under a diverse set of potential future scenarios (Snyder et al., 2006; Dong, 2006; Ben-Tal et al., 2009; Mulvey et al., 1995). We do not propose a concrete definition for robustness, but strategically view the robustness of a SCN as its ability to continue to meet demand under a wide ranging, but bounded, set of demand variations including occasional demand spikes.

The topic of SCN robustness, while nascent compared to SCN resilience, has been studied extensively in the last decade. Cardona-Valdés et al. (2011) present a seminal study examining a two-echelon supply chain incorporating demand uncertainty. They solve this model through the usage of a two-stage stochastic optimization model, where risk is modeled via scenarios. More recently, Keyvanshokooh et al. (2016) modeled a stochastic supply chain network design problem incorporating uncertainty in both cost and demand, and K1liç and Tuzkaya (2015) designed a SCN to maximize profit while handling demand uncertainty through the use of a two-stage stochastic optimization model using scenarios. Such approaches broadly mirror our own. However, the key difference is that our research considers both robustness to demand uncertainties and resilience to disruptions.
Developing a resilient, robust supply chain

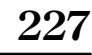




\section{JDAL} 5,2

228

\subsection{Resilience and robustness in tandem}

To the best of our knowledge, only three other papers present approaches to design a SCN, which is simultaneously resilient to disruption and incorporates scenario-based robust optimization. Jabbarzadeh et al. (2016) design a SCN where facilities can be "hardened" to reduce their potential for disruption. These hardened facilities supply lost demand from unreliable facilities that have experienced a disruption. The researchers further study effects of demand and supply fluctuations on the solution. In order to solve this problem, Jabbarzadeh et al. use a hybrid robust-stochastic optimization model, which they solve using a Lagrangian relaxation. In comparison, we do not examine resilience through hardening nodes but rather by establishing redundant routes. Sadghiani et al. (2015) examine resilience through a set-covering framework, where each demand node requires redundancy of supply. Robustness is integrated through the consideration of numerous scenarios. This approach is similar to our own, as we also utilize redundancy to handle resilience and multiple scenarios to handle robustness. The key differences which make our research unique are that our problem is solved as a network flow instead of as a set-covering problem and we establish a risk framework to guide the required amount of redundancy. Zhalechian et al. (2018) design a SCN using several resilience approaches, including design complexity and multiple allocations. They solve this problem using a two-stage stochastic optimization model, where the scenarios have been chosen using a scenario reduction technique. Our approach is broadly aligned with that proposed by Zhalechian, but we differ in that as we propose a riskanalysis framework for establishing constraints on the required resilience by node, and we focus more pragmatically on solving for a real world SCN.

\section{Risk analysis and model formulation}

In Figure 1, we provide a small-scale $\mathrm{SCN}$ example. $R_{i}$ represents the required resilience for each demand node $i$; equivalently, the number of supply nodes required to ensure that demand node $i$ has an acceptable level of risk associated with its demand not being met due to supply disruptions. The required resilience $R_{i}$ could be determined for each demand node $i$ through numerous decision-analysis frameworks. We provide a framework based on demand node location focused on conflict, infrastructure and climate risks. A resilience requirement score $R_{i}=1$ indicates an extremely stable location with solid infrastructure and temperate climate presenting a low risk of supply disruption. Nodes with a resilience requirement score of $R_{i}=2$ indicate locations with increased risk either due to conflict, infrastructure and climate or some combination thereof for which a single redundant supply node is deemed necessary to ensure the risk of supply disruptions is controlled. Higher $R_{i}$ values then 2 are also possible, each representing an increased level of required resilience in order to control risk of supply disruption.

The importance of demand robustness can also be visualized in Figure 1 . Consider that the normal demand pattern represented below is perturbed such that $M_{4}=5$ changes to $M_{4}=10$. This could be due to increased humanitarian operations (e.g. Ebola) in the local area. The

Figure 1. spoke SCN with two transshipment hubs in blue and four demand nodes in green. The maximum number of demand nodes each transshipment node can supply is three. The demand $M_{i}$ and the required resilience $R_{i}$ are also provided for each demand node

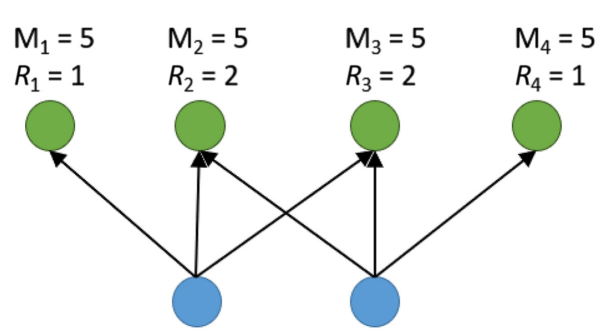


$\mathrm{SCN}$, as depicted, could struggle to respond to such a change, since the entirety of the change in demand would be borne by the transshipment node on the right. Conversely, if the normal demand pattern represented above is perturbed such that $M_{3}=5$ changes to $M_{3}=10$, the SCN may be able to more efficiently respond, as both transshipment nodes could contribute to fulfilling the demand change.

The supply chain in Western Africa is currently an efficient hub and spoke network. However, the focus on efficiency inhibits the network's ability to function in emergency or disaster situations (lack of resilience), as well as hinders its ability to function well under diverse scenarios with changed system parameters (lack of robustness). The ability to operate under both emergency and crisis situations is paramount in humanitarian and military operations. The need for this logistics network will remain far into the future. Our study considers design elements to ensure that both resilience and robustness are incorporated into the network supporting West Africa. This research develops a framework to identify and quantify risk in West Africa and then develops and optimizes a mathematical model to find the best routes to ensure necessary network resilience, and finally, this research uses stochastic analysis of plausible demand scenarios to ensure network robustness.

To address the logistical challenges present in West Africa - large distances, poor infrastructure, insecure routes, etc. - the supply chain was restructured to achieve a higherservice level and increased efficiency. The network operates under a hub-and-spoke design with a main hub, 6 transshipment nodes and 54 other potential demand locations. Any of the transshipment nodes, as well as the main hub, may also experience demand.

Table 1 shows the 16 West African countries that are included in the analysis, along with the number of potential demand nodes within each country. The European hub serves as a "dummy node," where supplies originate and ship to the main hub. The main hub of entry into West Africa and the other six transshipment locations were determined by decision-makers prior to the undertaking of this study. We seek to assess and increase the network's resilience by changing the transshipment hubs and adding backup links between these hubs and end demand nodes. A key difference in our approach is that we examine resilience from the perspective of link availability. This is a fundamentally different approach to prior resilience research that primarily focuses on node availability. We posit that link availability is the

\begin{tabular}{lc}
\hline Country & Number of nodes \\
\hline Benin & 1 \\
Burkina Faso* & 8 \\
Cameroon* & 5 \\
Chad & 4 \\
Ivory Coast & 1 \\
Gabon & 1 \\
Ghana & 4 \\
Liberia & 1 \\
Mali & 8 \\
Mauritania & 4 \\
Central African Republic & 1 \\
Niger* & 10 \\
Nigeria & 8 \\
Senegal* & 3 \\
Sierra Leone & 1 \\
Togo & 1 \\
Note(s): Countriesidentified as having
\end{tabular}

Note(s): Countries identified as having transshipment nodes in original architecture are indicated with a “*”
Developing a resilient, robust supply chain 
JDAL 5,2

230

primary concern for distribution systems in highly uncertain environments, such as West Africa, where large distances and risky areas must be traversed. Focusing on link availability enables a country-wide viewpoint for risk assessment and recognizes that over large distances, the links are much more vulnerable than the nodes.

\subsection{Risk framework}

The network configuration as first established does not implement any backup routes for material flow, possibly leaving nodes unsupported where an upstream hub, or route, disruption could occur. Though the existing air routes are flexible by nature, because of the long distances between locations and delays inherent to country clearance and border issues, a relatively fixed-delivery schedule has been implemented. Therefore, a transshipment node disruption would leave its supported nodes vulnerable for some time. The primary goal of this research is to identify an optimal variant of the network that adds resilience to disruption while maintaining efficiency and adding robustness to demand changes. Toward that end, our model requires each location be connected to more than one transshipment node. However, determining "appropriate" levels of redundancy is non-trivial, as these decisions necessitate a risk analysis framework and implementation - our first major contribution. We focus our risk analysis on three areas of interest for every country: internal conflict, infrastructure and terrain/climate.

Table 2 gives a short description of these three categories, along with the available scores and the meaning of each score by category. Classifications for conflict are determined by the U.S. Department of State Travel Advisory, and classifications for infrastructure are determined by the Central Intelligence Agency (CIA) World Fact Book (Central Intelligence Agency, 2018; U.S Department of State-Bureau of Consular affairs, 2018). Classification for terrain/climate was accomplished by the research team, thus presenting one subjective view. However, the classifications could be adjusted based on subject matter expertise or changing contextual conditions. Our unique approach incorporates the risk framework into the SCN design, establishing the value of the parameter $R_{i}$, which is used in our optimization model to generate a proposed resilient, robust and efficient supply chain for Western Africa.

Table 3 presents the results for each country. To determine aggregated risk, we place equal weight on each of three criteria and sum the individual scores to assess an overall (aggregated) risk score for each country. Such a framework does not preclude the application of non-equal weights if a subject matter expert determines one of the three categories to be more or less significant than the others.

Lastly, we compute a required resilience level, $R_{i}$, for each location $i$, which is based on the aggregated-risk score for that location's country. For the purposes of this research, the

Table 2.

Resilience risk analysis Score framework:

Description and scores for three considered categories of internal conflict, infrastructure and terrain/climate

\begin{tabular}{|c|c|c|c|c|}
\hline & & Conflict & Infrastructure & $\begin{array}{l}\text { Terrain/ } \\
\text { Climate }\end{array}$ \\
\hline Description & $\begin{array}{l}\text { Internal disputes and } \\
\text { drug and human } \\
\text { trafficking }\end{array}$ & $\begin{array}{l}\text { Air, land, and sea } \\
\text { capabilities }\end{array}$ & $\begin{array}{l}\text { Disruptions relating to } \\
\text { natural phenomena }\end{array}$ & \\
\hline \multirow[t]{3}{*}{ Score } & 0 & $\begin{array}{l}\text { Exercise normal } \\
\text { precaution when } \\
\text { traveling }\end{array}$ & $\begin{array}{l}\text { Ranked in Top 3rd in } \\
\text { World Fact Book }\end{array}$ & No issues \\
\hline & 1 & $\begin{array}{l}\text { Exercise increased } \\
\text { caution when traveling }\end{array}$ & $\begin{array}{l}\text { Ranked in Middle 3rd in } \\
\text { World Fact Book }\end{array}$ & Mild issues \\
\hline & 2 & $\begin{array}{l}\text { Do not travel/ } \\
\text { reconsider travel }\end{array}$ & $\begin{array}{l}\text { Ranked in bottom 3rd in } \\
\text { World Fact Book }\end{array}$ & $\begin{array}{l}\text { Severe } \\
\text { issues }\end{array}$ \\
\hline
\end{tabular}


following step function was used to assign required resilience levels $R_{i}$ based on the calculated aggregated risk. As noted in the description of Figure 1 lower $R_{i}$ scores indicate more stable geographical, geopolitical and climatic conditions requiring fewer backup supply arcs, while higher $R_{i}$ scores indicate increasingly less-stable regions requiring higher numbers of backup arcs to mitigate risks.

$$
R_{i}= \begin{cases}2 & \text { if Aggregated Risk }=0 \text { or } 1 \\ 3 & \text { if Aggregated Risk }=2 \text { or } 3 \\ 4 & \text { if Aggregated Risk } \geq 4\end{cases}
$$

\subsection{Mathematical formulation}

We developed an explicit mathematical formulation of the distribution network in West Africa as a two-staged stochastic, mixed-integer linear program in order to determine the optimal location for transshipment locations and route connections. The first stage determines transshipment node locations and the connections to demand nodes. The second stage solves the network flow problem, routing demand from transshipment nodes to demand nodes with the dual goals of minimizing transportation cost and maximizing resilience. Robustness to demand perturbations is informed by various subject matter experts and historically-informed acute emergency scenarios. To provide a precise statement of this problem, we define as follows:

Sets:

I Set of nodes, $i \in I=\{1, \ldots, 61\}$

$M \quad$ Set of transportation modes, $m \in M=\{1=$ air, $2=$ land, $3=$ sea $\}$

$S \quad$ Set of scenarios, $s \in S=\{1, \ldots, 10\}$

$K(i, j) \quad$ Set of available transportation modes between location $i$ and location $j . K(i, j) \in M$

\begin{tabular}{|c|c|c|c|c|c|c|c|}
\hline Country & Conflict & Infrastructure & $\begin{array}{l}\text { Terrain/Climate } \\
\text { Description }\end{array}$ & Score & $\begin{array}{l}\text { Aggregated } \\
\text { risk }\end{array}$ & $R_{i}$ & \\
\hline Benin & 0 & 2 & \multirow{4}{*}{$\begin{array}{l}\text { Volcano in W. Africa with } \\
\text { fatal levels of gas }\end{array}$} & 0 & 2 & 3 & \\
\hline Burkina Faso & 2 & 1 & & 0 & 3 & 3 & \\
\hline Cameroon & 1 & 1 & & 2 & 4 & 4 & \\
\hline $\begin{array}{l}\text { Central African } \\
\text { Republic }\end{array}$ & 2 & 1 & & 0 & 3 & 3 & \\
\hline Chad & 2 & 1 & \multirow{12}{*}{$\begin{array}{l}\text { Possible torrential flooding } \\
\text { during rainy season }\end{array}$} & 0 & 3 & 3 & Table 3. \\
\hline Gabon & 0 & 1 & & 0 & 1 & 2 & Resilience risk analysis \\
\hline Ghana & 0 & 0 & & 0 & 0 & 2 & by country: Explicit \\
\hline Ivory Coast & 1 & 0 & & 1 & 2 & 3 & $\begin{array}{l}\text { risk scores are first } \\
\text { assigned to each }\end{array}$ \\
\hline Liberia & 0 & 0 & & 0 & 0 & 2 & country by category, \\
\hline Mali & 2 & 1 & & 0 & 3 & 3 & $\begin{array}{l}\text { scores are then } \\
\text { summed toculate a }\end{array}$ \\
\hline Mauritania & 2 & 1 & & 0 & 3 & 3 & $\begin{array}{l}\text { summed to calculate an } \\
\text { agoregat risk metric. }\end{array}$ \\
\hline Niger & 2 & 1 & & 0 & 3 & 3 & and finally, a resilience \\
\hline Nigeria & 2 & 0 & & 0 & 2 & 3 & metric $R_{i}$ is assigned to \\
\hline Senegal & 0 & 1 & & 0 & 1 & 2 & each country based on \\
\hline Sierra Leone & 1 & 1 & & 1 & 3 & 3 & $\begin{array}{r}\text { its aggregated risk } \\
\text { according to }\end{array}$ \\
\hline Togo & 1 & 1 & & 0 & 2 & 3 & equation (1) \\
\hline
\end{tabular}

Developing a resilient, robust supply chain

231 
Parameters:

5,2

$U_{m}=$ Hourly operating cost of transportation mode $m$

$T_{i, j}^{m}=\quad$ Travel time from location $i$ to location $j$ using transportation mode $m$

$B$ ig $M=$ A large number, which is used for logical modeling

$V=\quad$ Number of transshipment node connections

$232 \quad R_{i}=\quad$ Required resilience at location $i$, and explicitly, the number of connections required at location $i$

$C_{i}=\quad$ Maximum number of connections established at location $i$

$G_{i}=\quad$ Fixed cost of establishing a hub at location $i$

$D_{i, s}=\quad$ Location $i$ demand under scenario $s$

$Q_{i, j}^{m}=\quad$ Cost for establishing a connection between location $i$ and location $j$ for transportation mode $m$

$P_{s}=\quad$ Probability of scenario $s$

Decision variables:

$x_{i}=\quad$ Binary variable; equals 1 if transshipment node $i$ is open and 0 otherwise.

$y_{i, j}^{m}=\quad$ Binary variable; equals 1 if a connection is established between transshipment location $i$ and location $j$ using transportation mode $m$ and 0 otherwise.

$w_{i, j}^{m, s}=\quad$ Binary variable; equals 1 if established connection $y_{i, j}^{m}$ is used under scenario $s$ and 0 otherwise.

$z_{i, j}^{k}=\quad$ Quantity (short tons (STONS)) shipped from location $i$ to location $j$ using connection $w_{i, j}^{m, s}$

The network allows material to be delivered using three methods of transportation as follows: air, land and sea. Our underlying network is assumed fully connected for air and land transport modes, while sea connections exist between all nodes located on the coasts, with travel times between two nodes calculated as follows: for any two locations $i, j \in I$, we compute the estimated travel time $T_{i, j}^{m}$ for transportation mode $m$ based on the distance between two nodes and the average speed of the mode. If a transportation mode is infeasible between two locations, that specified route receives an input of BigM, ensuring the model does not select an infeasible route. Since a core tenant of the network is a single point of entry from Europe, the initial version of the model established as infeasible all other routes to West Africa.

Travel time by air was calculated with the Euclidean distance formula using each location's latitude and longitude coordinates as determined by Google Earth. Distances were then multiplied by the average speed of 335 miles per hour (mph) - the average speed of an L100 - which is the primary cargo aircraft currently in use in the region. Only pairs of locations with airports and runways within 20 miles of each endpoint had calculated air distances. Shared airports between two locations were precluded. Ownership of an airport was determined by closest distance. As this model is predominately interested in minimizing cost and unmet demand vs minimizing time, loading/unloading times were not included.

Ground transport times between locations were estimated using the recommended path from Google Maps and were only assigned if the route linking the pair of locations could be traversed by land vehicles. Due to poor road quality and traffic, the planning factor currently in use is $25 \mathrm{mph}$. Specifically, land transportation was deemed unsuitable if another form of transportation was also required, e.g. both ferry and ground transportation.

Finally, sea transportation times were calculated using the website, Sea-Distance.org, which provides nautical miles between ports (sea-distances.org, 2019). For consistency, the calculated nautical miles were then converted to miles. Sea distances were only computed for pairs of locations with sea ports at each endpoint. The estimated sea transport speed is 28 mph. We assume that there is no capacity limit for each mode of transportation. While any 
individual aircraft has capacity limits, we assume that sufficient transport vehicles exist to meet cargo demand by any modality; an assumption in line with the relatively sparse demand patterns experienced in the region.

The problem of transshipment node selection, network connections establishment, mode choice and inventory distribution is formulated by the mixed integer linear program as follows, which we call the African Supply Chain Network Problem (ASCNP):

$$
\begin{gathered}
\min _{\boldsymbol{w}, \boldsymbol{x}, \boldsymbol{y}, \boldsymbol{z}} \sum_{i \in I} G_{i} x_{i}+\sum_{i \in I} \sum_{j \in I} \sum_{m \in K(i, j)} Q_{i, j}^{m} y_{i, j}^{m}+\sum_{s \in S} \sum_{i \in I} \sum_{j \in I} \sum_{m \in K(i, j)} T_{i, j}^{m} U_{m} P_{s} w_{i, j}^{m, s} \\
\text { s.t. } \quad \sum_{i \in I} x_{i}=V, \\
y_{i, j}^{m}<=x_{i} \quad \forall i, j \in I, i \neq j, \quad \forall m \in M, \\
\sum_{m} \sum_{i, i \neq j} y_{i, j}^{m}>=R_{j} \quad \forall j \in I, \\
\sum_{m} \sum_{j, j \neq i} y_{i, j}^{m}<=C_{i} x_{i} \quad \forall i \in I, \\
\sum_{i, j}^{m, s}<=y_{i, j}^{m} \quad \forall i, j \in I, i \neq j, \quad \forall m \in M, \quad \forall s \in S, \\
z_{i, j}^{m, s}<=B i g M w_{i, j}^{m, s} \quad \forall i, j \in I, i \neq j \quad \forall m \in M, \\
\sum_{j, i}^{m, s}-\sum_{m} \sum_{j, j \neq i} z_{i, j}^{m, s}>=D_{i, s} \quad \forall i \in I, \quad \forall s \in S .
\end{gathered}
$$

ASCNP is structured as a two-stage, multi-objective optimization problem. The first stage seeks to minimize hub and link establishment and the expected transportation cost. The second stage seeks to maximize network resilience by minimizing complexity, criticality, density and expected unmet demand. The first term in the objective function (2) is the investment costs for establishing transshipment nodes. This represents the cost for establishing the capability of a node to serve as an air, land or sea transshipment point. The cost would include both physical infrastructure and administrative support. The second term in the objective function is the investment costs for establishing links between the nodes. The link costs are mode specific and would also include infrastructure, equipment and administrative functions. The third term in the objective function is the expected operational costs. These costs are a function of serving demand and are determined by the travel time between two nodes, given the mode that is selected and the cost of that mode. 
JDAL 5,2
While travel time is an imperfect metric, attributing accurate costs for land, air and sea shipments was beyond scope of this research, so transit time multiplied by mode cost per hour is used as a cost proxy.

Constraint (3) sets the required number of transshipment nodes. There are seven transshipment nodes (the main hub plus six extant transshipment hubs) in the base model in accordance with the real world. This number is varied during succeeding model runs. Constraint (4) ensures that a link can only be established between nodes $i$ and $j$ if a transshipment node is established at $i$. Constraint (5) specifies the number of transshipment node connections, $R_{j}$, which a location $j$ requires. This ensures network resilience is commensurate with location risk and is determined according to the methodology in section 3.1. Constraint (6) imposes an upper bound, $C$, on the number of outbound connections a transshipment node can support $\left(x_{i}=1\right)$. Our initial assumption is that all transshipment nodes can support the same number of locations, but we cap the total number of locations that a transshipment node can support at 30 and thus $C=30$ for our base case. Constraint (7) ensures that no material can flow between locations $i$ and $j$ under scenario $s$ unless a connection is established between them. Constraint (8) imposes an upper bound on the quantity of material sent between $i$ and $j$ using mode $m$. Constraint (9) represents the flow constraint at location $i$ and ensures that the net flow of material to a location meets its demand (i.e. total flow of material delivered to a location minus total flow of material shipped out from that location is, at least, equal to its demand, $D_{i, s}$ ). As a result, locations can accumulate excess material, which is an occurrence not atypical of real world instances of warehouses having a surplus of material to insulate network from demand spikes.

\section{Scenario-based robustness analysis}

The goal of this research is to design a resilient and robust network for West Africa to cover 61 potential demand locations. Resilience to potential network disruptions is handled in the ASCNP model via the $R_{i}$ parameter as informed by the risk assessment framework developed in section 3.1. The resilience of the resulting network is ensured by requiring that each location be serviced by a calculated number of transshipment facilities. Locations with higher risk of disruption due to conflict, infrastructure or terrain have higher-connection requirements. Hence, in the event of a network disruption, a previously identified and established backup route could be activated, preventing demand shortages. Identifying backup routes early ensures that contracts and contingency plans can be enacted.

The establishment of new transshipment hubs and backup routes will have an associated cost. Hub setup costs are selected by random draw from U(100, 400) (thousands US\$). Recognizing that transportation modes will have different infrastructure and support requirements, link setup costs are also selected by random draw from air: $U(20,40)$, land: $U(5,10)$ and sea: $U(15,30)$.

However, network disruptions are not the only risk that a supply network needs to be able to handle. Scenario-based robustness analysis was used to assess how changing demand signals and/or capacity restrictions resulted in changes in the network design. This was accomplished by analyzing different demand scenarios and network modifications. These demand scenarios and network modifications are presented below.

\subsection{Scenarios considered}

The West Africa logistics network is a low-demand, high-priority network. Its inherent low demand induces a high-natural variability throughout the year as a result of a lack of "smoothing." Also, the region of West Africa is also prone to instability and emergency situations both natural and man-made. Developing a supply chain that insulates against 
these acute perturbations ensures military and governmental ability to respond to these emergencies. The following scenarios are based on history and subject matter expertise; however, the probabilities and real-world demand distributions have been replaced with nominal values for operational security considerations. In order to preserve operational security, all demand and risk data presented in this research have been sanitized. Real-world data are available upon request to authorized agencies.

We assume that demand at each location is uniformly distributed, and we assign each node's demand using a random draw from $U(10,50)$ (thousands STONS). Due to various instability issues in West Africa, demand is prone to fluctuations. To account for this variation, we incorporated three scenarios based on their historical occurrences: epidemic, terrorist attack and internal conflict. For each of the scenarios, we associate a probability of occurrence as well as the resulting percentage increase in demand to account for the heightened requirement for food, medical supplies, equipment and personnel. Table 4 summarizes each demand scenario's affected countries, likelihood of occurring and percentage increase in demand.

As multiple crisis scenarios could occur simultaneously, there are eight possible combinations of crises scenarios, including a "nothing happens" scenario. Using Bayes' theorem, we can determine the probabilities for all eight possible combinations of crisis. The resulting eight possible crisis scenarios are detailed in Table 5 . There are seven possible location scenarios (one 1country, three 2-country and three 3-country scenarios) for each event occurrence, and thus, 512 total $\left(8^{3}\right)$ possible scenarios are there, including the no event occurrence scenario.

We assume that an event occurrence can affect all three countries with probability 0.4 , each of the three pairs of countries with probability 0.15 and each country with probability 0.05 . Further, we assume that in case a country is affected by an event, all of its locations will be affected as well. Using the epidemic event and its corresponding countries, namely Liberia, Senegal and Sierra Leone as an example, we assign a probability of 0.4 in case all three countries are affected, a probability of 0.15 for each of the country pairs (Liberia and Senegal), (Liberia and Sierra Leone) and (Senegal and Sierra Leone) and a probability of 0.05 for each of Liberia, Senegal and Sierra Leone. The same probability assignment scheme applies for the events of terrorist attack and internal conflict. The risk probabilities could be easily changed and the model re-run with inputs from subject matter experts. We chose higher probabilities

\begin{tabular}{llll}
\hline & Epidemic & Terrorist attack & Internal conflict \\
\hline Likelihood & $20 \%$ & $50 \%$ & $80 \%$ \\
Countries & Liberia, Senegal and Sierra & Chad, Niger and & Burkina Faso, Mali and \\
affected & Leone & Nigeria & Niger \\
Demand increase & $300 \%$ & $200 \%$ & $100 \%$
\end{tabular}

Source(s): www.cdc.gov (2017), Roby (2018)
Developing a resilient, robust supply chain

Table 4.

Three non-independent crisis scenarios considered for robustness analysis along with probabilities of occurrence, locations effected and associated increase in regional demand to handle crisis

\begin{tabular}{|c|c|c|c|c|c|}
\hline Event notation & Epidemic & Terrorist attack & Internal conflict & Prob. Occurrence & \\
\hline None & & & & $8 \%$ & \\
\hline A & 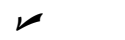 & & & $2 \%$ & Table 5 \\
\hline $\mathrm{B}$ & & $\nu$ & & $8 \%$ & $\begin{array}{r}1 \text { able } 5 . \\
\text { Ejoht possible }\end{array}$ \\
\hline $\mathrm{C}$ & & & & $32 \%$ & $\begin{array}{l}\text { Eignt possidie } \\
\text { combinations of the }\end{array}$ \\
\hline $\mathrm{AB}$ & $\nu$ & レ & & $2 \%$ & three crisis scenarios \\
\hline $\mathrm{AC}$ & レ & & $\nu$ & $8 \%$ & with associated \\
\hline $\mathrm{BC}$ & & レ & レ & $32 \%$ & probability of \\
\hline $\mathrm{ABC}$ & $\nu$ & $\nu$ & $\nu$ & $8 \%$ & occurrence \\
\hline
\end{tabular}


JDAL 5,2

236 for multiple-country events under the assumption that there would be cross-border spillover in the event of one of the occurrences.

The large number of scenarios makes the ASCNP computationally infeasible. As a result, we employ a scenario-reduction technique to reduce the number of scenarios the model considers. This technique consists of first-ranking scenario probabilities in descending order. The top $N$ scenarios are selected and then the probabilities of the selected scenarios are normalized. Given the number of selected scenarios, the ASCNP model selects the transshipment hubs, the demand nodes each transshipment hub supports (capped at 30) as well as the transportation mode used on each link and backup links. The objective function minimizes the cost of operating the network, given the possible scenarios. Accounting for $N=10$ scenarios is computationally feasible and provides enough coverage of the design space to ensure the highest-risk scenarios are accounted for. The top-ten scenarios as found by our scenario reduction technique are shown in Table 6.

\subsection{Number of hubs sensitivity analysis}

In addition to the scenario-specific demand changes resulting from the crisis events described above, we expand upon the base model by conducting sensitivity analysis on the number of transshipment hubs in the network (Constraint 3). Because the number of hubs is a fixed parameter, we recognize that the number chosen will have a substantial impact on both cost and resilience. We run the model with $5,6,7,8,10$ and 15 transshipment hubs. This set brackets the real world situation of seven transshipment hubs as well as expands outward to explore how several additional transshipment hubs would impact the SCN. The establishment of hubs and links will take time and resources, but fewer hubs will result in less-possible backup connections, i.e. less resilience. Therefore, varying the number of hubs either validates the current network configuration or provides recommended modifications for how the ASCNP could improve robustness and resilience at the lowest possible cost.

\section{Findings and contributions}

The AFSCN model selects transshipment hubs, assigns demand nodes to each transshipment hub and establishes an optimal flow by transportation mode. Out of these tasks, arguably the most interesting, to the decision-makers, is an examination of the recommended transshipment nodes along with their aggregate demand connections. This information enables the decision-makers to ensure they have both invested in the correct transshipment hubs and have invested enough to right-size each transshipment hub. Table 7 shows the

\begin{tabular}{lc}
\hline Description & Normalized probability \\
\hline Internal conflict in all three countries & 0.2599 \\
No events & 0.1622 \\
Terrorist attack and internal conflict in all three countries & 0.1039 \\
Internal conflict in Burkina Faso and Mali & 0.0972 \\
Internal conflict in Burkina Faso and Niger & 0.0972 \\
Internal conflict in Mali and Niger & 0.0972 \\
Terrorist attack in all three countries & 0.0650 \\
Terrorist attack in Chad and Niger & 0.0391 \\
Plus internal conflicts in all three countries & 0.0391 \\
Terrorist attack in Chad and Nigeria & \\
Plus internal conflicts in all three countries & 0.0391 \\
Terrorist attack in Niger and Nigeria & \\
Plus internal conflicts in all three countries &
\end{tabular}

Table 6.

The top-ten most likely scenarios out of 512 possible scenarios 


\begin{tabular}{|c|c|c|c|c|c|c|c|}
\hline \multirow[b]{2}{*}{ Transshipment hub location } & \multicolumn{6}{|c|}{ Number of connections per transshipment hub } & \multirow{2}{*}{$\begin{array}{l}\text { Developing a } \\
\text { resilient, robust }\end{array}$} \\
\hline & 5 hubs & 6 hubs & 7 hubs & 8 hubs & 10 hubs & 15 hubs & \\
\hline Accra & - & 30 & 28 & 27 & 23 & 18 & \\
\hline Agadez & _- & - & - & - & 16 & - & \\
\hline Арара & - & - & 30 & 21 & - & 14 & \\
\hline Cotonou & - & - & - & - & - & 11 & \\
\hline Djibo & - & 30 & 30 & 28 & 27 & 18 & 237 \\
\hline Freetown & - & 30 & 27 & 22 & 163 & 15 & \\
\hline Kaduna & - & - & 30 & - & 18 & 13 & \\
\hline Kano & - & 30 & 26 & 20 & 17 & 12 & \\
\hline Lome & - & - & - & - & 21 & 15 & \\
\hline Maroua & - & 30 & - & 30 & 23 & 17 & \\
\hline Menaka & - & - & - & - & - & 12 & \\
\hline Mouhoun & - & - & - & - & - & 3 & \\
\hline Sokoto & - & - & - & 24 & - & 11 & \\
\hline Tessalit & - & - & - & - & - & 3 & \\
\hline Tichit & - & 29 & 8 & 7 & 5 & 4 & \\
\hline Timbuktu & - & - & - & - & 13 & 8 & \\
\hline Yearly cost (thousands US\$) & - & 371,072 & 370,936 & 370,992 & 371,176 & 371,685 & \\
\hline $\begin{array}{l}\text { Note(s): The numbers in tal } \\
\text { this transshipment node was } \\
\text { set-up }\end{array}$ & $\begin{array}{l}\text { re a cour } \\
\text { establish }\end{array}$ & $\begin{array}{l}\text { arcs out } \\
\text { ot the case }\end{array}$ & $\begin{array}{l}\text { ach estab } \\
\text { uestion. } B\end{array}$ & $\begin{array}{l}\text { ed transsh } \\
\text { m row is y }\end{array}$ & $\begin{array}{l}\text { nent node. } \\
\text { ly cost of }\end{array}$ & $\begin{array}{l}\text { indicates } \\
\text { ation and }\end{array}$ & $\begin{array}{r}\text { Vertical axis is set of all } \\
\text { experienced } \\
\text { transshipment hubs }\end{array}$ \\
\hline
\end{tabular}

recommended locations for each transshipment hub option and the number of connections for each location. The total number of connections is the same across all architectures.

Given the demands and constraints for AFSCN, there is no feasible solution if we only allow five transshipment hubs. Six transshipment hubs is feasible, but this architecture is actually more expensive than when we allow seven transshipment hubs - the solution currently employed in West Africa. Our recommended hubs are not the exact match as those in the actual network, but they are geographically similar. There are two items of note as we move to eight or more transshipment hubs. First, as we would expect in a hub and spoke network, the cost of each solution increases with the number of hubs once a certain threshold is reached. After seven transshipment hubs, the network cost is increased by the non-trivial cost of establishing additional transshipment hubs. There is an optimal size from a cost perspective.

Second, there is a strong pattern that as the number of transshipment hubs is increased, new transshipment hubs are added to the existing nodes. There are, however, important exceptions. Eight transshipment hubs do not include Kaduna, which in the case of seven transshipment hubs was maxed out at 30 connections. This follows traditional portfolio optimization results in which an optimal portfolio using a subset of the total funds rarely is an exact subset of the optimal portfolio using the full set of funds. However, in the context of supply chain design, it is an issue we would bring to the decision-makers, as they may want to develop a distribution network aimed at growing toward eight transshipment hubs, thus selecting a sub-optimal design at six or seven transshipment hubs. Switching hubs may not be a feasible option given the required investment costs.

In addition to suggesting the optimal set of transshipment hubs that meet resilience requirements at the lowest possible cost, the model suggests connections between transshipment hubs and down-range demand nodes. These results represent both primary and backup connections that would need to be contractually established to ensure the required resilience. Table 8 provides a sample of this output with 20 of the 61 demand nodes for the 6 transshipment hub scenario. Similar tables were produced for all other scenarios. 
JDAL

5,2

\section{8}

Table 8.

Suggested connections between

transshipment hubs (top row), a

representative subset of 20 of the 61 demand sites (first column) and A 1 in a cell indicates a suggested connection between a

transshipment hub and a demand site by modality

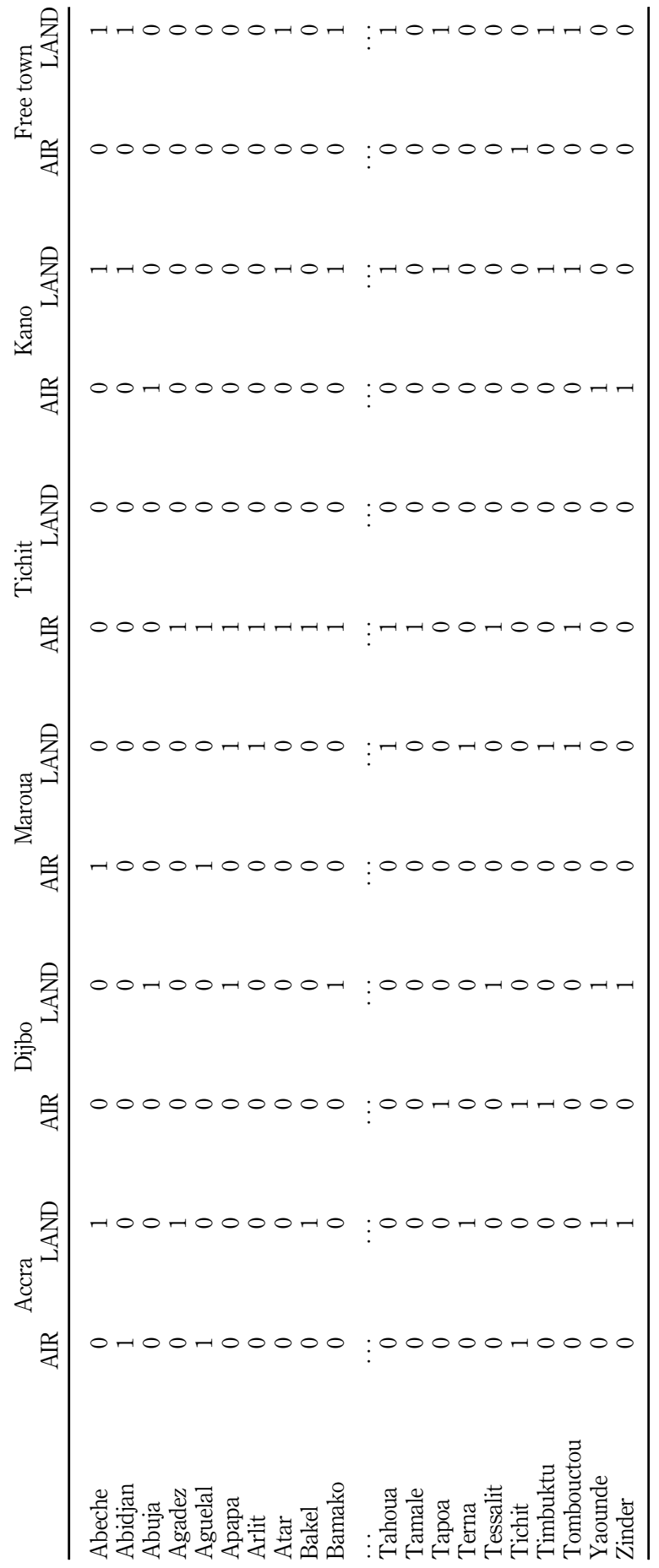


This research provides decision-makers insight into how both resilience and robustness can be accounted for in real world networks. It also highlights the tradeoffs that managers must be cognizant of when balancing cost against resilience and robustness. Given the total number of primary and backup connections is held constant across the different hub designs, a more decentralized network, i.e. more hubs, would indicate more resilience even though the total number of connections are the same. This is the argument for choosing an increased-hub design even though it may be more expensive. Or decision-makers have the option of selecting the least expensive design that meets the resilience and robustness requirements given the risk assessments. A key contribution is that the options are clearly presented.

Academically, the main contribution of this paper is a method by which supply chain designers and network planners can integrate risk assessments, local capabilities and environmental factors into facility location decisions. We provide a risk analysis framework that classifies levels of conflict, infrastructure capability and environmental risk. These factors are generalizable across any facility location problem and are relevant to all supply chains. We then show how publicly available data sets - provided by the U.S. State Department and CIA Factbook - can be used to populate the risk framework with location-specific assessments. We used a scenario-reduction technique to focus on the highest-risk scenarios. The number of possible situations is extremely large in a real, complex environment. This method highlights an approach to focus on those situations that are highest risk, highest cost or any other area of focus that is deemed most critical. The countries of West Africa and real-demand scenarios are used to demonstrate how these factors are taken into account to design an efficient network that is both resilient to disruption and robust to demand changes. To the best of our knowledge, this is the first such effort to do this.

\section{Conclusion and future research}

This research study created a resilient operational road map to allow a network to function during disruptions. Our AFSCN model incorporates 61 West African demand locations and suggests transshipment nodes by scenario. To account for demand fluctuations due to disruption - terrorist attacks, epidemics and/or natural disaster - multiple different scenario occurrences and their likelihood were identified. We then analyzed the resulting network connections and shipping flow.

There is a cost to establish backup connections for resilience and robustness. Specifically, such connections lead to solutions that use less efficient routes. In fact, when accounting for the distances of connections to transshipment nodes, the model does not always choose the cheapest shipping connection for a single connection but solves for the cheapest architecture overall. Two key points emerge from this insight. First, there is an efficiency cost to resilience. Modeling the network allows us to determine that cost, which can be captured as we saw in Table 7. Second, resilience must be considered at the supply chain level and not the individual node level. As our AFSCN model demonstrates, the optimal system design may lead to suboptimal solutions for individual nodes and routes.

This study opens several opportunities for future research. The number of items shipped by air, land and sea is unconstrained. However, in real-world situations, different locations have different capacity and means available, e.g. air crew sizes and number of land/sea vehicles. A more realistic instance of this model would be to impose a limit on the quantity of material that can shipped by each mode. Finally, robustness in this model is handled by examining the highest likelihood scenarios through a two-stage stochastic optimization problem. A more rigorous, and presently open question, would be to incorporate resilience into a robust optimization framework.
Developing a resilient, robust supply chain 


\section{JDAL} 5,2

\section{References}

An, Y., Zhang, Y. and Zeng, B. (2015), "The reliable hub-and-spoke design problem: models and algorithms", Transportation Research Part B: Methodological, Vol. 77, pp. 103-122.

Ben-Tal, A., El Ghaoui, L. and Nemirovski, A. (2009), Robust Optimization, Princeton University Press.

Berdica, K. (2002), "An introduction to road vulnerability: what has been done, is done and should be done", Transport Policy, Vol. 9 No. 2, pp. 117-127.

Borgström, B. (2005), "Exploring efficiency and effectiveness in the supply chain: a conceptual analysis", Proceedings from the 21st IMP Conference, Rotterdam, p. 22.

Campbell, J.F. (1996), "Hub location and the p-hub median problem", Operations Research, Vol. 44 No. 6, pp. 923-935.

Cardona-Valdés, Y., Álvarez, A. and Ozdemir, D. (2011), "A bi-objective supply chain design problem with uncertainty”, Transportation Research Part C: Emerging Technologies, Vol. 19 No. 5, pp. 821-832.

Central Intelligence Agency (2018), “Central intelligence agency: world fact book”, available at: https:// www.cia.gov/library/publications/the-world-factbook/geos/gh.html.

Chowdhury, M.M.H. and Quaddus, M. (2017), "Supply chain resilience: conceptualization and scale development using dynamic capability theory", International Journal of Production Economics, Vol. 188, pp. 185-204.

Daskin, M.S. (2011), Network and Discrete Location: Models, Algorithms, and Applications, John Wiley \& Sons.

Dong, M. (2006), "Development of supply chain network robustness index", International Journal of Services Operations and Informatics, Vol. 1 Nos 1-2, pp. 54-66.

Heydari, B., Mosleh, M. and Dalili, K. (2015), "Efficient network structures with separable heterogeneous connection costs", Economics Letters, Vol. 134, pp. 82-85.

Hutchison, D. and Sterbenz, J.P. (2018), “Architecture and design for resilient networked systems", Computer Communications, Vol. 131, pp. 13-21.

Jabbarzadeh, A., Fahimnia, B., Sheu, J.-B. and Moghadam, H.S. (2016), "Designing a supply chain resilient to major disruptions and supply/demand interruptions", Transportation Research Part B: Methodological, Vol. 94, pp. 121-149.

Jackson, M.O. and Wolinsky, A. (1996), “A strategic model of social and economic networks”, Journal of Economic Theory, Vol. 71 No. 1, pp. 44-74.

Keyvanshokooh, E., Ryan, S.M. and Kabir, E. (2016), "Hybrid robust and stochastic optimization for closed-loop supply chain network design using accelerated benders decomposition”, European Journal of Operational Research, Vol. 249 No. 1, pp. 76-92.

Kılıç, Y.E. and Tuzkaya, U.R. (2015), “A two-stage stochastic mixed-integer programming approach to physical distribution network design”, International Journal of Production Research, Vol. 53 No. 4, pp. 1291-1306.

Kim, Y., Chen, Y.-S. and Linderman, K. (2015), "Supply network disruption and resilience: a network structural perspective", Journal of Operations Management, Vol. 33, pp. 43-59.

Klibi, W., Martel, A. and Guitouni, A. (2010), "The design of robust value-creating supply chain networks: a critical review", European Journal of Operational Research, Vol. 203 No. 2, pp. 283-293.

Matisziw, T.C., Murray, A.T. and Grubesic, T.H. (2009), "Exploring the vulnerability of network infrastructure to disruption", The Annals of Regional Science, Vol. 43 No. 2, p. 307.

Mattsson, L.-G. and Jenelius, E. (2015), "Vulnerability and resilience of transport systems-a discussion of recent research", Transportation Research Part A: Policy and Practice, Vol. 81, pp. 16-34.

Mensah, P. and Merkuryev, Y. (2014), "Developing a resilient supply chain”, Procedia-Social and Behavioral Sciences, Vol. 110, pp. 309-319. 
Mirchandani, P.B. and Francis, R.L. (1990), Discrete Location Theory, John Wiley \& Sons, New York.

Muckensturm, J.R. and Longhorn, D.C. (2019), "Assessing the vulnerability of military theater distribution routes", Journal of Defense Analytics and Logistics, Vol. 3 No. 1, pp. 60-82.

Mulvey, J.M., Vanderbei, R.J. and Zenios, S.A. (1995), "Robust optimization of large-scale systems", Operations Research, Vol. 43 No. 2, pp. 264-281.

Ng, A.K., Jiang, C., Larson, P., Prentice, B. and Duval, D.T. (2018), Transport Nodal System, Elsevier.

Reggiani, A., Nijkamp, P. and Lanzi, D. (2015), "Transport resilience and vulnerability: the role of connectivity”, Transportation Research Part A: Policy and Practice, Vol. 81, pp. 4-15.

Roby, C. (2018), "5 African crises to watch in 2018", available at: https://www.devex.com/news/ 5african-crises-to-watch-in-2018-91836.

Rostami, B., Kämmerling, N., Buchheim, C. and Clausen, U. (2018), "Reliable single allocation hub location problem under hub breakdowns", Computers and Operations Research, Vol. 96, pp. 15-29.

Sadghiani, N.S., Torabi, S. and Sahebjamnia, N. (2015), "Retail supply chain network design under operational and disruption risks", Transportation Research Part E: Logistics and Transportation Review, Vol. 75, pp. 95-114.

sea-distances.org (2019), "Sea distances/port distances", available at: https://sea-distances.org.

Sheffi, Y. and Rice, J.B. Jr (2005), "A supply chain view of the resilient enterprise", MIT Sloan Management Review, Vol. 47 No. 1, p. 41.

Snyder, L.V., Scaparra, M.P., Daskin, M.S. and Church, R.L. (2006), "Planning for disruptions in supply chain networks", in Models, Methods, and Applications for Innovative Decision Making, INFORMS, pp. 234-257.

U.S Department of State-Bureau of Consular affairs (2018), "Travel.state.gov", available at: https:// travel.state.gov/content/travel/en/international-travel.html.

Wagner, S.M. and Neshat, N. (2010), "Assessing the vulnerability of supply chains using graph theory", International Journal of Production Economics, Vol. 126 No. 1, pp. 121-129.

www.cdc.gov (2017), "Ebola (Ebola Virus Disease): 2014-2016 Ebola outbreak distribution in West Africa, Technical Report, Centers for Disease Control and Prevention, available at: https://www. cdc.gov/vhf/ebola/history/2014-2016-outbreak/distribution-map.html.

Yang, K., Yang, L. and Gao, Z. (2017), "Hub-and-spoke network design problem under uncertainty considering financial and service issues: a two-phase approach”, Information Sciences, Vol. 402 , pp. 15-34.

Zhalechian, M., Torabi, S.A. and Mohammadi, M. (2018), "Hub-and-spoke network design under operational and disruption risks", Transportation Research Part E: Logistics and Transportation Review, Vol. 109, pp. 20-43.

\section{Corresponding author}

Bruce A. Cox can be contacted at: bruceacox1@gmail.com

For instructions on how to order reprints of this article, please visit our website:

www.emeraldgrouppublishing.com/licensing/reprints.htm

Or contact us for further details: permissions@emeraldinsight.com 